Sebastian Baran (Kraków)

ZBigniew PALMOWsKi (Wrocław)

\title{
OPTIMIZING THE EXPECTED UTILITY OF DIVIDEND PAYMENTS FOR A CRAMÉR-LUNDBERG RISK PROCESS
}

Abstract. We consider the problem of maximizing the discounted utility of dividend payments of an insurance company whose reserves are modeled as a classical Cramér-Lundberg risk process. We investigate this optimization problem under the constraint that the dividend rate is bounded. We prove that the value function satisfies the Hamilton-Jacobi-Bellman equation and we identify the optimal dividend strategy.

1. Introduction. The problem of finding optimal dividend strategies for an insurance company has been studied since De Finetti [3]. The continuous time risk process was studied for the first time in the seminal work of Gerber [4]. He assumed that the reserve process $R=\left(R_{t}\right)_{t \geq 0}$ of an insurance company is modeled by a classical Cramér-Lundberg risk process,

$$
R_{t}=x+\mu t-\sum_{i=1}^{N_{t}} Y_{i},
$$

where $Y_{1}, Y_{2}, \ldots$ are i.i.d positive random variables with absolutely continuous d.f. $F_{Y}$ representing the claims, $N=\left(N_{t}\right)_{t \geq 0}$ is an independent Poisson process with intensity $\lambda>0$ modeling the times at which the claims occur, $x \geq 0$ denotes the initial surplus and $\mu$ is a premium intensity. We consider the above Poisson process $N$ and the sequence $\left\{Y_{i}, i \in \mathbb{N}\right\}$ on a common probability space $(\Omega, \mathcal{F}, \mathbb{P})$.

For the classical dividend problem, apart from the reserve process (1.1), we consider the dividend payments. Let $C=\left(C_{t}\right)_{t \geq 0}$ be an adapted and

2010 Mathematics Subject Classification: Primary 60K10; Secondary 93E20.

Key words and phrases: stochastic control, Hamilton-Jacobi-Bellman equation, dividend problem, capital injection, utility function.

Received 3 February 2017; revised 14 April 2017.

Published online 24 August 2017. 
nondecreasing process representing all accumulated dividend payments up to time $t$. Then the regulated process $X=\left(X_{t}\right)_{t \geq 0}$ is given by

$$
X_{t}=R_{t}-C_{t} .
$$

We observe the regulated process $X_{t}$ until the time of ruin

$$
\tau=\inf \left\{t \geq 0: X_{t}<0\right\} .
$$

Obviously the time of the ruin of an insurance company depends on the dividend strategy, and after the ruin occurs, no dividends are paid. As usual we assume that the net profit condition $\mu>\lambda E\left(Y_{1}\right)$ for the Cramér-Lundberg risk process is fulfilled.

For the classical dividend problem we define the value of the dividends by

$$
v_{C}(x)=\mathbb{E}_{x}\left(\int_{0}^{\tau} e^{-\beta t} d C_{t}\right),
$$

where $\beta>0$ is a discount factor, $\mathbb{E}_{x}$ means expectation with respect to $\mathbb{P}_{x}(\cdot)=P\left(\cdot \mid X_{0}=x\right)$, and the value function by

$$
v(x)=\sup _{C \in \mathrm{C}} v_{C}(x),
$$

with $\mathrm{C}$ being the set of all admissible accumulated dividend strategies $\left(C_{t}\right)_{t \geq 0}$.

In the mathematical finance and actuarial literature, there is a good deal of work being done on dividend barrier models and the problem of finding an optimal policy of paying dividends - see Schmidli [11] for an overview. In this paper we assume additionally that $\left(C_{t}\right)_{t \geq 0}$ is absolutely continuous with respect to the Lebesgue measure (see e.g. Hubalek and Schachermayer [6]). Then the process $C$ admits a density process denoted by $c=\left(c_{t}\right)_{t \geq 0}$, modeling the intensity of the dividend payments in continuous time, that is, for $t \geq 0$,

$$
C_{t}=\int_{0}^{t} c_{s} d s \quad \text { a.s. }
$$

Then we can consider the discounted cumulative utility of dividend payments

$$
v_{c}(x)=\mathbb{E}_{x}\left(\int_{0}^{\tau} e^{-\beta t} U\left(c_{t}\right) d t\right),
$$

where $U$ is some fixed utility function, and the value function equals

$$
v(x)=\sup _{c \in \mathfrak{C}} v_{c}(x),
$$

with $\mathfrak{C}$ being the set of all admissible strategies $\left(c_{t}\right)_{t \geq 0}$.

A dividend density process $\left(c_{t}\right)_{t \geq 0}$ is admissible if it is a nonnegative, adapted and càdlàg process and no dividend is payed after ruin occurs: $c_{t}=0$ for all $t \geq \tau$. As noted in [11, Rem. 2.1], we choose càdlàg strategies instead 
of more often used left-continuous ones. We study this optimization problem under the constraint that only dividend strategies with dividend rate bounded by a fixed constant are admissible, i.e. $0 \leq c_{t} \leq c_{0}<\infty$ for all $t \geq 0$ and some fixed number $c_{0}>0$. Finally, we assume that ruin cannot be caused by dividend payment.

Under this set-up we prove that the value function is differentiable and it solves the Hamilton-Jacobi-Bellman (HJB) equation.

2. Some properties of the value function. Recall that the regulated process is given by

$$
X_{t}=x+\mu t-\sum_{i=1}^{N_{t}} Y_{i}-\int_{0}^{t} c_{s} d s
$$

and the value function is defined in 1.4 .

From now on we assume that $U: \mathbb{R}_{\geq 0} \rightarrow \mathbb{R}_{\geq 0}$ is continuous, nonnegative, strictly increasing, strictly concave and $U(0)=0$.

LEMma 2.1. The optimal value function $v(x)$ is nonnegative and bounded by $U\left(c_{0}\right) / \beta$ and converges to $U\left(c_{0}\right) / \beta$ a.s. as $x \rightarrow \infty$. Furthermore, $v(x)$ is increasing, Lipschitz continuous and therefore absolutely continuous.

Proof. Using the same strategy for two initial capitals shows that $v$ is increasing. Since $U$ is nonnegative, $v$ is nonnegative. Clearly, $v(x) \leq$ $\int_{0}^{\infty} U\left(c_{0}\right) e^{-\beta t} d t=U\left(c_{0}\right) / \beta$. Consider the strategy $c^{*}=\left(c_{t}^{*}\right)$ which pays dividends at constant rate $c_{0}$ for all $t \geq 0$ and consider the ruin time $\tau_{x}^{c^{*}}$ associated with the strategy $c^{*}$ and the initial capital $x$. Then

$$
v(x) \geq \mathbb{E}_{x}\left(\int_{0}^{\tau_{x}^{c^{*}}} e^{-\beta t} U\left(c_{0}\right) d t\right)=\left(1-\mathbb{E}_{x}\left[e^{-\beta \tau_{x}^{c^{*}}}\right]\right) \frac{U\left(c_{0}\right)}{\beta} .
$$

We now show that $\tau_{x}^{c^{*}}$ converges to infinity almost surely as $x \rightarrow \infty$. Choose $y \geq x$ and one realization $\omega \in \Omega$ of $\sum_{i=1}^{N_{t}} Y_{i}$. We will denote it by adding a superscript $\omega$ to the relevant objects. Since

$$
y+\left(\mu-c_{0}\right) t-\sum_{i=1}^{N_{t}^{\omega}} Y_{i}^{\omega} \geq x+\left(\mu-c_{0}\right) t-\sum_{i=1}^{N_{t}^{\omega}} Y_{i}^{\omega},
$$

it follows that $\tau_{y}^{c^{*}} \geq \tau_{x}^{c^{*}}$, i.e. the function $x \mapsto \tau_{x}^{c^{*}}$ is nondecreasing for fixed $\omega$. Thus $\tau_{x}^{c^{*}}$ has either a finite or an infinite limit. Without loss of generality we can consider the regulated risk process $X$ stopped at the ruin time. If we assume that there exists $\omega$ such that the limit $\tau^{c^{*}}$ of the $\tau_{x}^{c^{*}}$ is finite then we get a contradiction: in this case we can always find $x$ large 
enough to get

$$
x+\left(\mu-c_{0}\right) \tau^{c^{*}}-\sum_{i=1}^{N_{\tau^{*}}^{\omega}} Y_{i}^{\omega}>0 .
$$

Hence $\tau_{x}^{c^{*}}$ converges to infinity a.s. as $x \rightarrow \infty$.

By bounded convergence the quantity $\mathbb{E}_{x}\left[e^{-\beta \tau_{x}^{c^{*}}}\right]$ appearing in $(2.1)$ converges to zero as $x \rightarrow \infty$. Therefore $v(x)$ converges to $U\left(c_{0}\right) / \beta$ as $x \rightarrow \infty$.

Let us now prove that $v(x)$ is absolutely continuous. Let $y>x \geq 0$. We denote by $c=\left(c_{t}\right)$ a strategy for the initial capital $y$. We now take the strategy $\bar{c}=\left(\bar{c}_{t}\right)$ that starts at the initial reserve $x$, pays no dividends if $X_{t}^{\bar{c}}<y$ and follows the strategy $c$ after reaching level $y$. The strategy $\bar{c}$ is of course admissible. In the event of no claims, the reserve process $X_{t}^{\bar{c}}$ reaches $y$ at time $t_{0}=(y-x) / \mu$. Since the probability that it reaches $y$ before the first claim (i.e. $\left.P\left(T_{1}>t_{0}\right)\right)$ is $e^{-\lambda t_{0}}$, we get

$$
v(x) \geq v_{\bar{c}}(x) \geq P\left(T_{1}>t_{0}\right) e^{-\beta t_{0}} v(y)=e^{-(\lambda+\beta)(y-x) / \mu} v(y) .
$$

Since $v$ is increasing and bounded, we get

$$
\begin{aligned}
0 & \leq v(y)-v(x) \leq v(y)-e^{-(\lambda+\beta)(y-x) / \mu} v(y) \\
& =v(y)\left(1-e^{-(\lambda+\beta)(y-x) / \mu}\right) \leq \frac{U\left(c_{0}\right)}{\beta}(\lambda+\beta) \frac{y-x}{\mu} .
\end{aligned}
$$

Thus $v$ is Lipschitz continuous on $[0, \infty)$, and so differentiable almost everywhere in $[0, \infty)$. By Lipschitz continuity on compact sets, the above calculations give bounds for the density $v^{\prime}(x)$, which is therefore integrable on compact sets. This implies that $v$ is absolutely continuous (see for example Wheeden and Zygmund [13]).

3. The Hamilton-Jacobi-Bellman equation. In the proof of the main theorem we will need a lemma that constructs in a measurable way nearly optimal strategies for all initial states from a compact subset of $\mathbb{R}_{+}$. The proofs of this lemma and of the following theorem are based on the proofs of [11, Theorem 2.32] and [2, Theorem 3.2].

Lemma 3.1. For all $x \in[a, b] \subset[0, \infty)$ one can find, in a measurable way, a strategy $\hat{c}(x)$ such that $v_{\hat{c}(x)}(x) \geq v(x)-\varepsilon$.

Proof. Choose $\varepsilon>0$ and let $n \in \mathbb{N}$ be large enough that

$$
\frac{U\left(c_{0}\right)(\lambda+\beta)}{\beta \mu} \cdot \frac{b-a}{n} \leq \frac{\varepsilon}{2} .
$$

Let $x_{k}=(k / n) b+(1-k / n) a$ for $0 \leq k \leq n$. For each $x_{k}$ there is a strategy $\hat{c}\left(x_{k}\right)=\left(\hat{c}_{t}\left(x_{k}\right)\right)$ such that $v_{\hat{c}\left(x_{k}\right)}\left(x_{k}\right)>v\left(x_{k}\right)-\varepsilon / 2$. Now we construct a strategy for all $x \in[a, b]$. For each $x \in[a, b]$ there exist $k_{0}$ such that 
$x_{k_{0}} \leq x<x_{k_{0}+1}$. We choose $\hat{c}(x)=\hat{c}\left(x_{k_{0}}\right)=\left(\hat{c}_{t}\left(x_{k_{0}}\right)\right)$, i.e. the same strategy as for $x_{k_{0}}$. Then

$$
v_{\hat{c}(x)}(x)=v_{\hat{c}\left(x_{k_{0}}\right)}(x) \geq v_{\hat{c}\left(x_{k_{0}}\right)}\left(x_{k_{0}}\right) \geq v\left(x_{k_{0}}\right)-\varepsilon / 2 .
$$

From 2.2, 3.1 and the definition of $x_{k_{0}}$ we get

$$
\begin{aligned}
v\left(x_{k_{0}}\right) & \geq v(x)-\frac{U\left(c_{0}\right)(\lambda+\beta)}{\beta \mu}\left(x-x_{k_{0}}\right) \\
& \geq v(x)-\frac{U\left(c_{0}\right)(\lambda+\beta)}{\beta \mu} \cdot \frac{b-a}{n} \geq v(x)-\varepsilon / 2 .
\end{aligned}
$$

This shows that for all $x \in[a, b]$ we can find, in a measurable way, a strategy $\hat{c}(x)$ such that $v_{\hat{c}(x)}(x) \geq v(x)-\varepsilon$.

In the following theorem we derive the Hamilton-Jacobi-Bellman equation and prove that the value function $v(x)$ is smooth enough.

THEOREM 3.2. If $c_{0}<\mu$, then the optimal value function $v(x)$ is continuously differentiable and satisfies the Hamilton-Jacobi-Bellman equation

$$
\sup _{0 \leq c \leq c_{0}}\left\{(\mu-c) v^{\prime}(x)-(\beta+\lambda) v(x)+U(c)+\lambda \int_{0}^{x} v(x-y) d F_{Y}(y)\right\}=0 .
$$

Proof. Since $v$ is Lipschitz continuous and increasing, it is differentiable almost everywhere. Moreover, at the points where $v$ is not differentiable, it is differentiable from the left and from the right.

Let $h>0$ and fix $c \in\left[0, c_{0}\right]$. Consider now the following strategy:

$$
c_{t}= \begin{cases}c & \text { for } 0 \leq t \leq T_{1} \wedge h, \\ \hat{c}_{t-\left(T_{1} \wedge h\right)}\left(X_{T_{1} \wedge h}\right) & \text { for } t>T_{1} \wedge h,\end{cases}
$$

where $\hat{c}(x)$ is the nearly optimal strategy defined in Lemma 3.1. The first claim $T_{1}$ happens with density $\lambda e^{-\lambda h}$, and $T_{1}$ is larger than $h$ with probability $e^{-\lambda h}$. Thus

$$
\begin{aligned}
& v(x) \geq e^{-\lambda h}\left[\int_{0}^{h} U(c) e^{-\beta t} d t+e^{-\beta h} v_{\hat{c}}(x+(\mu-c) h)\right]+\int_{0}^{h}\left[\int_{0}^{t} U(c) e^{-\beta s} d s\right. \\
& \left.+e^{-\beta t} \int_{0}^{x+(\mu-c) t} v_{\hat{c}}(x+(\mu-c) t-y) d F_{Y}(y)\right] \lambda e^{-\lambda t} d t \\
& \geq e^{-\lambda h}\left[\int_{0}^{h} U(c) e^{-\beta t} d t+e^{-\beta h} v(x+(\mu-c) h)\right]+\int_{0}^{h}\left[\int_{0}^{t} U(c) e^{-\beta s} d s\right. \\
& \left.+e^{-\beta t} \int_{0}^{x+(\mu-c) t} v(x+(\mu-c) t-y) d F_{Y}(y)\right] \lambda e^{-\lambda t} d t-\varepsilon .
\end{aligned}
$$


The constant $\varepsilon$ is arbitrary, so we can let it tend to zero. Rearranging the terms and dividing by $h$ yields

$$
\begin{aligned}
& 0 \geq \frac{v(x+(\mu-c) h)-v(x)}{h}-\frac{1-e^{-(\beta+\lambda) h}}{h} v(x+(\mu-c) h) \\
&+e^{-\lambda h} \frac{1}{h} \int_{0}^{h} U(c) e^{-\beta t} d t+\frac{1}{h} \int_{0}^{h}\left[\int_{0}^{t} U(c) e^{-\beta s} d s\right. \\
&\left.\quad+e^{-\beta t} \int_{0}^{x+(\mu-c) t} v(x+(\mu-c) t-y) d F_{Y}(y)\right] \lambda e^{-\lambda t} d t .
\end{aligned}
$$

Now we choose a strategy $c(h)=\left(c_{t}(h)\right)$ such that $v_{c}(x) \geq v(x)-h^{2}$. Note that $c_{t}(h)$ is just the $c_{t}$ defined in (3.4). Let $a(t)=\mu t-\int_{0}^{t} c_{s}(h) d s$. Then

$$
\begin{aligned}
v(x) \leq & h^{2}+v_{c}(x)=h^{2}+e^{-\lambda h}\left[\int_{0}^{h} U\left(c_{t}(h)\right) e^{-\beta t} d t+e^{-\beta h} v_{c}(x+a(h))\right] \\
& +\int_{0}^{h}\left[\int_{0}^{t} U\left(c_{t}(h)\right) e^{-\beta s} d s+e^{-\beta t} \int_{0}^{x+a(t)} v_{c}(x+a(t)-y) d F_{Y}(y)\right] \lambda e^{-\lambda t} d t .
\end{aligned}
$$

Since $v_{c}(x) \leq v(x)$, it follows that

$$
\begin{aligned}
v(x) \leq & h^{2}+e^{-\lambda h}\left[\int_{0}^{h} U\left(c_{t}(h)\right) e^{-\beta t} d t+e^{-\beta h} v(x+a(h))\right] \\
& +\int_{0}^{h}\left[\int_{0}^{t} U\left(c_{t}(h)\right) e^{-\beta s} d s+e^{-\beta t} \int_{0}^{x+a(t)} v(x+a(t)-y) d F_{Y}(y)\right] \lambda e^{-\lambda t} d t .
\end{aligned}
$$

Rearranging the terms and dividing by $h$ yields

$$
\begin{aligned}
0 \leq & h+\frac{v(x+a(h))-v(x)}{h}-\frac{1-e^{-(\beta+\lambda) h}}{h} v(x+a(h)) \\
+e^{-\lambda h} \frac{1}{h} \int_{0}^{h} U\left(c_{t}(h)\right) e^{-\beta t} d t & +\frac{1}{h} \int_{0}^{h}\left[\int_{0}^{t} U\left(c_{t}(h)\right) e^{-\beta s} d s\right. \\
& \left.+e^{-\beta t} \int_{0}^{x+a(t)} v(x+a(t)-y) d F_{Y}(y)\right] \lambda e^{-\lambda t} d t .
\end{aligned}
$$

Since $e^{-\beta t} \leq 1$ for $t \geq 0$, and $U$ is nonnegative and strictly concave, from Jensen's inequality it follows that

$$
\frac{1}{h} \int_{0}^{h} U\left(c_{t}(h)\right) e^{-\beta t} d t \leq \frac{1}{h} \int_{0}^{h} U\left(c_{t}(h)\right) d t \leq U\left(\frac{1}{h} \int_{0}^{h} c_{t}(h) d t\right) .
$$


Therefore

$$
\begin{aligned}
0 \leq & h+\frac{v(x+a(h))-v(x)}{h}-\frac{1-e^{-(\beta+\lambda) h}}{h} v(x+a(h)) \\
+e^{-\lambda h} U\left(\frac{1}{h} \int_{0}^{h} c_{t}(h) d t\right) & +\frac{1}{h} \int_{0}^{h}\left[\int_{0}^{t} U\left(c_{t}(h)\right) e^{-\beta s} d s\right. \\
& \left.+e^{-\beta t} \int_{0}^{x+a(t)} v(x+a(t)-y) d F_{Y}(y)\right] \lambda e^{-\lambda t} d t .
\end{aligned}
$$

Note that

$$
0 \leq \int_{0}^{h} c_{t}(h) d t \leq \int_{0}^{h} c_{0} d t=c_{0} h
$$

Thus $a(h) \rightarrow 0$ as $h \rightarrow 0^{+}$. Since $v$ is continuous, it follows that

$$
\lim _{h \rightarrow 0^{+}} \frac{1-e^{-(\beta+\lambda) h}}{h} v(x+a(h))=(\beta+\lambda) v(x)
$$

and

$$
\lim _{h \rightarrow 0^{+}} \frac{1}{h} \int_{0}^{h} e^{-\beta t}\left(\int_{0}^{x+a(t)} v(x+a(t)-y) d F_{Y}(y)\right) \lambda e^{-\lambda t} d t=\lambda \int_{0}^{x} v(x-y) d F_{Y}(y) .
$$

Since $c_{t}$ are bounded $U$ is nonnegative and continuous, and since $0 \leq e^{-\beta s} \leq 1$ for all $s \geq 0$, we have

$$
0 \leq \frac{1}{h} \int_{0}^{h}\left(\int_{0}^{t} U\left(c_{t}(h)\right) e^{-\beta s} d s\right) \lambda e^{-\lambda t} d t \leq \frac{1}{h} \int_{0}^{h}\left(\int_{0}^{t} U\left(c_{0}\right) d s\right) \lambda d t=\frac{\lambda U\left(c_{0}\right)}{2} h .
$$

Therefore,

$$
\lim _{h \rightarrow 0^{+}} \frac{1}{h} \int_{0}^{h}\left(\int_{0}^{t} U\left(c_{t}(h)\right) e^{-\beta s} d s\right) \lambda e^{-\lambda t} d t=0 .
$$

Thus, with the exception of the second and fourth terms, the terms on the right-hand side of (3.6) converge.

Consider now the second term. We have

$$
\frac{v(x+a(h))-v(x)}{h}=\frac{v(x+a(h))-v(x)}{a(h)} \cdot \frac{a(h)}{h} .
$$

Note that $h^{-1} \int_{0}^{h} c_{t}(h) d t \in\left[0, c_{0}\right]$. Thus there exists a sequence $h_{n} \rightarrow 0^{+}$ such that

$$
\lim _{h_{n} \rightarrow 0^{+}} \frac{1}{h_{n}} \int_{0}^{h_{n}} c_{t}\left(h_{n}\right) d t=\tilde{c}
$$


for some $\tilde{c} \in\left[0, c_{0}\right]$. Note that $\tilde{c}$ might be a random variable as for now. However, we will show later that there exists exactly one nonrandom value in $\left[0, c_{0}\right]$ which realizes the supremum in the HJB equation (3.7). Hence

$$
\lim _{h_{n} \rightarrow 0^{+}} \frac{a\left(h_{n}\right)}{h_{n}}=\mu-\tilde{c} .
$$

Since $U$ is continuous, it follows that

$$
\lim _{h_{n} \rightarrow 0^{+}} U\left(\frac{1}{h_{n}} \int_{0}^{h_{n}} c_{t}\left(h_{n}\right) d t\right)=U(\tilde{c})
$$

Recall that $c_{0}<\mu$, hence $a(h)>0$ and

$$
\lim _{h \rightarrow 0^{+}} \frac{v(x+a(h))-v(x)}{a(h)}=v_{+}^{\prime}(x),
$$

where $v_{+}^{\prime}(x)$ means the right derivative (which is finite as mentioned above). Thus, for the sequence $h_{n} \rightarrow 0^{+}$chosen as above, (3.6) implies that

$$
0 \leq(\mu-\tilde{c}) v_{+}^{\prime}(x)-(\beta+\lambda) v(x)+U(\tilde{c})+\lambda \int_{0}^{x} v(x-y) d F_{Y}(y) .
$$

Consider now inequality (3.5). Since $c \leq c_{0}<\mu$, it follows that $(\mu-c) h>0$ for all $c \in\left[0, c_{0}\right]$, and as a consequence

$$
\lim _{h \rightarrow 0^{+}} \frac{v(x+(\mu-c) h)-v(x)}{(\mu-c) h}=v_{+}^{\prime}(x) .
$$

Thus, for all $c \in\left[0, c_{0}\right]$, from 3.5 we have

$$
0 \geq(\mu-c) v_{+}^{\prime}(x)-(\beta+\lambda) v(x)+U(c)+\lambda \int_{0}^{x} v(x-y) d F_{Y}(y) .
$$

This means that for $c=\tilde{c}$,

$$
0=(\mu-\tilde{c}) v_{+}^{\prime}(x)-(\beta+\lambda) v(x)+U(\tilde{c})+\lambda \int_{0}^{x} v(x-y) d F_{Y}(y)
$$

and $v_{+}^{\prime}(x)$ satisfies the Hamilton-Jacobi-Bellman equation

$$
\sup _{0 \leq c \leq c_{0}}\left\{(\mu-c) v_{+}^{\prime}(x)-(\beta+\lambda) v(x)+U(c)+\lambda \int_{0}^{x} v(x-y) d F_{Y}(y)\right\}=0
$$

provided $c_{0}<\mu$. We can repeat the argument for any possible limit of

$$
\lim _{h_{n} \rightarrow 0^{+}} \frac{1}{h_{n}} \int_{0}^{h_{n}} c_{t}\left(h_{n}\right) d t
$$

leading to the same equation (3.7). This means that (3.7) is satisfied for any $\omega \in \Omega$. Formally, the choice of $\tilde{c}$ depends on $\omega$, and the value of the 
above limit may also depend on the sequence $h_{n} \rightarrow 0$. However, we will show below that there exists exactly one nonrandom value on $\left[0, c_{0}\right]$ which realizes the supremum in (3.7). In order to distinguish limits, we denote the corresponding limit by $\hat{c}$. Note that the function $\zeta(c):=-c v_{+}^{\prime}(x)+U(c)$ is strictly concave in $\left[0, c_{0}\right]$ because the sum of a strictly concave function and a linear function is strictly concave. Since $\zeta$ is strictly concave, it follows that it has only one local maximum. Therefore $\tilde{c}=\hat{c}$. Thus, there exists exactly one limit $\tilde{c}$ which realizes the supremum in (3.7).

Assume that $h$ is small enough that $(x-(\mu-c) h) \wedge(x-a(h))>0$. Let us point out that $a(h)$ depends on the initial state. Therefore the $a(h)$ that is used from now on is different from the one defined in the previous part of the proof. Moreover, since $c_{t}(h)$ depends on the initial capital and we let $h \rightarrow 0$ in the further part of the proof, we make use of the measurable construction of nearly optimal strategies from Lemma 3.1. For the initial capital $x-(\mu-c) h$ inequality (3.5) yields

$$
\begin{aligned}
0 \geq & \frac{v(x-(\mu-c) h)-v(x)}{-h}-\frac{1-e^{-(\beta+\lambda) h}}{h} v(x) \\
& +e^{-\lambda h} \frac{1}{h} \int_{0}^{h} U(c) e^{-\beta t} d t+\frac{1}{h} \int_{0}^{h}\left[\int_{0}^{t} U(c) e^{-\beta s} d s\right. \\
& \left.+e^{-\beta t} \int_{0}^{x-(\mu-c)(h-t)} v(x-(\mu-c)(h-t)-y) d F_{Y}(y)\right] \lambda e^{-\lambda t} d t .
\end{aligned}
$$

As above, we conclude that

$$
0 \geq(\mu-c) v_{-}^{\prime}(x)-(\beta+\lambda) v(x)+U(c)+\lambda \int_{0}^{x} v(x-y) d F_{Y}(y)
$$

for all $c \in\left[0, c_{0}\right]$, where $v_{-}^{\prime}(x)$ means the left derivative (which is finite as mentioned above). For the initial capital $x-a(h)$ inequality (3.6) yields

$$
\begin{aligned}
0 \leq & +\frac{v(x-a(h))-v(x)}{-h}-\frac{1-e^{-(\beta+\lambda) h}}{h} v(x) \\
& +e^{-\lambda h} U\left(\frac{1}{h} \int_{0}^{h} c_{t}(h) d t\right)+\frac{1}{h} \int_{0}^{h}\left[\int_{0}^{t} U\left(c_{t}(h)\right) e^{-\beta s} d s\right. \\
& \left.+e^{-\beta t} \int_{0}^{x-a(h)+a(t)} v(x-a(h)+a(t)-y) d F_{Y}(y)\right] \lambda e^{-\lambda t} d t .
\end{aligned}
$$

Then there exists $\hat{c} \in\left[0, c_{0}\right]$ such that

$$
0 \leq(\mu-\hat{c}) v_{-}^{\prime}(x)-(\beta+\lambda) v(x)+U(\hat{c})+\lambda \int_{0}^{x} v(x-y) d F_{Y}(y) .
$$

Hence $v_{-}^{\prime}(x)$ satisfies the Hamilton-Jacobi-Bellman equation 
(3.12) $\sup _{0 \leq c \leq c_{0}}\left\{(\mu-c) v_{-}^{\prime}(x)-(\beta+\lambda) v(x)+U(c)+\lambda \int_{0}^{x} v(x-y) d F_{Y}(y)\right\}=0$, and as above there exists exactly one limit $\hat{c}$ which realizes the supremum in 3.12 .

Now we prove that $v$ is differentiable $\left(v_{+}^{\prime}(x)=v_{-}^{\prime}(x)\right)$. We know that $v_{+}^{\prime}$ and $v_{-}^{\prime}$ satisfy (3.7) and (3.12) respectively. We also know that there exist values $\tilde{c}$ and $\hat{c}$ such that the suprema in (3.7) and (3.12) respectively are attained. Thus

$$
(\mu-\tilde{c}) v_{+}^{\prime}(x)+U(\tilde{c})=(\mu-\hat{c}) v_{-}^{\prime}(x)+U(\hat{c}) .
$$

Since the supremum in 3.12 is attained at $\hat{c}$, it follows that

$$
(\mu-\hat{c}) v_{-}^{\prime}(x)+U(\hat{c}) \geq(\mu-\tilde{c}) v_{-}^{\prime}(x)+U(\tilde{c}) .
$$

Then (3.13) combined with (3.14) gives

$$
(\mu-\tilde{c}) v_{+}^{\prime}(x)+U(\tilde{c}) \geq(\mu-\tilde{c}) v_{-}^{\prime}(x)+U(\tilde{c}),
$$

and therefore

$$
(\mu-\tilde{c})\left(v_{+}^{\prime}(x)-v_{-}^{\prime}(x)\right) \geq 0 .
$$

Since $\tilde{c}<\mu$, this immediately leads to

$$
v_{+}^{\prime}(x) \geq v_{-}^{\prime}(x) .
$$

On the other hand, since the supremum in 3.7 is attained at $\tilde{c}$, it follows that

$$
(\mu-\tilde{c}) v_{+}^{\prime}(x)+U(\tilde{c}) \geq(\mu-\hat{c}) v_{+}^{\prime}(x)+U(\hat{c}) .
$$

Then, as above, we get

$$
v_{-}^{\prime}(x) \geq v_{+}^{\prime}(x) .
$$

This means that $v$ is differentiable, and $\tilde{c}=\hat{c}$. Summarizing, we have proved that there exist exactly one optimal value $c^{*}$ that simultaneously realizes the suprema in (3.7) and (3.12), and that the function $v$ is differentiable and satisfies the Hamilton-Jacobi-Bellman equation

$$
\sup _{0 \leq c \leq c_{0}}\left\{(\mu-c) v^{\prime}(x)-(\beta+\lambda) v(x)+U(c)+\lambda \int_{0}^{x} v(x-y) d F_{Y}(y)\right\}=0
$$

when $c_{0}<\mu$. This completes the proof.

THEOREM 3.3. If $c_{0}>\mu$ and $U$ is differentiable at $\mu$, then the optimal value function $v(x)$ is differentiable and satisfies the Hamilton-JacobiBellman equation

$$
\sup _{0 \leq c \leq c_{0}}\left\{(\mu-c) v^{\prime}(x)-(\beta+\lambda) v(x)+U(c)+\lambda \int_{0}^{x} v(x-y) d F_{Y}(y)\right\}=0 .
$$

If the supremum in (3.16) is not attained at $\mu$ then $v(x)$ is continuously differentiable. 
Proof. The proof is similar to that of Theorem 3.2 with some significant modifications.

Since $v$ is Lipschitz continuous and increasing, it is differentiable almost everywhere. Moreover, at the points where $v$ is not differentiable, it is differentiable from the left and from the right.

Let $h>0$ and fix $c \in\left[0, c_{0}\right]$. If $x=0$, we suppose that $c \leq \mu$, and if $x>0$, we let $h$ be small enough that $x+(\mu-c) h \geq 0$, i.e., ruin does not occur because of dividend payments. In the same way as in the above proof we can derive the inequality

$$
\begin{aligned}
& 0 \geq \frac{v(x+(\mu-c) h)-v(x)}{h}-\frac{1-e^{-(\beta+\lambda) h}}{h} v(x+(\mu-c) h) \\
&+e^{-\lambda h} \frac{1}{h} \int_{0}^{h} U(c) e^{-\beta t} d t+\frac{1}{h} \int_{0}^{h}\left[\int_{0}^{t} U(c) e^{-\beta s} d s\right. \\
&\left.\quad+e^{-\beta t} \int_{0}^{x+(\mu-c) t} v(x+(\mu-c) t-y) d F_{Y}(y)\right] \lambda e^{-\lambda t} d t .
\end{aligned}
$$

From 3.17) we have

$$
0 \geq(\mu-c) v_{+}^{\prime}(x)-(\beta+\lambda) v(x)+U(c)+\lambda \int_{0}^{x} v(x-y) d F_{Y}(y)
$$

for $c<\mu$. Moreover,

$$
0 \geq(\mu-c) v_{-}^{\prime}(x)-(\beta+\lambda) v(x)+U(c)+\lambda \int_{0}^{x} v(x-y) d F_{Y}(y)
$$

for $c>\mu$, and

$$
0 \geq-(\beta+\lambda) v(x)+U(c)+\lambda \int_{0}^{x} v(x-y) d F_{Y}(y)
$$

for $c=\mu$.

Now we choose a strategy $c(h)=\left(c_{t}(h)\right)$ such that $v_{c}(x) \geq v(x)-h^{2}$. Let $a(t)=\mu t-\int_{0}^{t} c_{s}(h) d s$. Then in the same way as in the proof of Theorem 3.2 we can derive the inequality

$$
\begin{aligned}
0 \leq & h+\frac{v(x+a(h))-v(x)}{h}-\frac{1-e^{-(\beta+\lambda) h}}{h} v(x+a(h)) \\
& +e^{-\lambda h} U\left(\frac{1}{h} \int_{0}^{h} c_{t}(h) d t\right)+\frac{1}{h} \int_{0}^{h}\left[\int_{0}^{t} U\left(c_{t}(h)\right) e^{-\beta s} d s\right. \\
& \left.+e^{-\beta t} \int_{0}^{x+a(t)} v(x+a(t)-y) d F_{Y}(y)\right] \lambda e^{-\lambda t} d t .
\end{aligned}
$$


Since $h^{-1} \int_{0}^{h} c_{t}(h) d t \in\left[0, c_{0}\right]$, there exists a sequence $h_{n} \rightarrow 0^{+}$such that

$$
\lim _{h_{n} \rightarrow 0^{+}} \frac{1}{h_{n}} \int_{0}^{h_{n}} c_{t}\left(h_{n}\right) d t=\tilde{c} \text { and } \lim _{h_{n} \rightarrow 0^{+}} \frac{a\left(h_{n}\right)}{h_{n}}=\mu-\tilde{c} .
$$

From inequality (3.21) we have

$$
0 \leq(\mu-\tilde{c}) v_{+}^{\prime}(x)-(\beta+\lambda) v(x)+U(\tilde{c})+\lambda \int_{0}^{x} v(x-y) d F_{Y}(y)
$$

if $\tilde{c}<\mu$. Furthermore,

$$
0 \leq(\mu-\tilde{c}) v_{-}^{\prime}(x)-(\beta+\lambda) v(x)+U(\tilde{c})+\lambda \int_{0}^{x} v(x-y) d F_{Y}(y)
$$

if $\tilde{c}>\mu$, and

$$
0 \leq-(\beta+\lambda) v(x)+U(\tilde{c})+\lambda \int_{0}^{x} v(x-y) d F_{Y}(y)
$$

if $\tilde{c}=\mu$.

Note that we do not know if $\tilde{c}$ is greater than, less than or maybe equal to $\mu$. We do not know if there is just one $\tilde{c}$ either. It is possible that more than one $\tilde{c}$ satisfies $(3.22),(3.23)$ or (3.24). At the moment we only know that (3.22), (3.23) or (3.24) is satisfied by at least one $\tilde{c}$.

Now we assume that $h$ is small enough that $(x-(\mu-c) h) \wedge(x-a(h))>0$. Note that the $a(h)$ here is different from the $a(h)$ defined in the previous part of the proof because we consider a different initial capital. For the initial capital $x-(\mu-c) h$, the inequality (3.17) gives

$$
\begin{aligned}
0 \geq & \frac{v(x-(\mu-c) h)-v(x)}{-h}-\frac{1-e^{-(\beta+\lambda) h}}{h} v(x) \\
& +e^{-\lambda h} \frac{1}{h} \int_{0}^{h} U(c) e^{-\beta t} d t+\frac{1}{h} \int_{0}^{h}\left[\int_{0}^{t} U(c) e^{-\beta s} d s\right. \\
& \left.+e^{-\beta t} \int_{0}^{x-(\mu-c)(h-t)} v(x-(\mu-c)(h-t)-y) d F_{Y}(y)\right] \lambda e^{-\lambda t} d t .
\end{aligned}
$$

As above, we get $(3.20)$ for $c=\mu$, and

$$
0 \geq(\mu-c) v_{-}^{\prime}(x)-(\beta+\lambda) v(x)+U(c)+\lambda \int_{0}^{x} v(x-y) d F_{Y}(y)
$$

for $c<\mu$. Moreover,

$$
0 \geq(\mu-c) v_{+}^{\prime}(x)-(\beta+\lambda) v(x)+U(c)+\lambda \int_{0}^{x} v(x-y) d F_{Y}(y)
$$

for $c>\mu$. 
Thus from (3.18), 3.27) and (3.20) we see that for all $c \in\left[0, c_{0}\right]$,

$$
0 \geq(\mu-c) v_{+}^{\prime}(x)-(\beta+\lambda) v(x)+U(c)+\lambda \int_{0}^{x} v(x-y) d F_{Y}(y),
$$

and from 3.19), 3.26) and 3.20 we find that for all $c \in\left[0, c_{0}\right]$,

$$
0 \geq(\mu-c) v_{-}^{\prime}(x)-(\beta+\lambda) v(x)+U(c)+\lambda \int_{0}^{x} v(x-y) d F_{Y}(y) .
$$

This means that

$$
\sup _{0 \leq c \leq c_{0}}\left\{(\mu-c) v_{+}^{\prime}(x)-(\beta+\lambda) v(x)+U(c)+\lambda \int_{0}^{x} v(x-y) d F_{Y}(y)\right\} \leq 0
$$

and

$$
\sup _{0 \leq c \leq c_{0}}\left\{(\mu-c) v_{-}^{\prime}(x)-(\beta+\lambda) v(x)+U(c)+\lambda \int_{0}^{x} v(x-y) d F_{Y}(y)\right\} \leq 0 .
$$

Now, for the initial capital $x-a(h)$ inequality (3.21) yields

$$
\begin{aligned}
0 \leq & h+\frac{v(x-a(h))-v(x)}{-h}-\frac{1-e^{-(\beta+\lambda) h}}{h} v(x) \\
& +e^{-\lambda h} U\left(\frac{1}{h} \int_{0}^{h} c_{t}(h) d t\right)+\frac{1}{h} \int_{0}^{h}\left[\int_{0}^{t} U\left(c_{t}(h)\right) e^{-\beta s} d s\right. \\
& \left.+e^{-\beta t} \int_{0}^{x-a(h)+a(t)} v(x-a(h)+a(t)-y) d F_{Y}(y)\right] \lambda e^{-\lambda t} d t .
\end{aligned}
$$

Since $h^{-1} \int_{0}^{h} c_{t}(h) d t \in\left[0, c_{0}\right]$, there exists a sequence $h_{n} \rightarrow 0^{+}$such that

$$
\lim _{h_{n} \rightarrow 0^{+}} \frac{1}{h_{n}} \int_{0}^{h_{n}} c_{t}\left(h_{n}\right) d t=\hat{c} \quad \text { and } \quad \lim _{h_{n} \rightarrow 0^{+}} \frac{a\left(h_{n}\right)}{h_{n}}=\mu-\hat{c} .
$$

Note that $\hat{c}$ may be different from $\tilde{c}$.

From (3.30) we get (3.24) if $\hat{c}=\mu$, and

$$
0 \leq(\mu-\hat{c}) v_{-}^{\prime}(x)-(\beta+\lambda) v(x)+U(\hat{c})+\lambda \int_{0}^{x} v(x-y) d F_{Y}(y)
$$

if $\hat{c}<\mu$. Furthermore,

$$
0 \leq(\mu-\hat{c}) v_{+}^{\prime}(x)-(\beta+\lambda) v(x)+U(\hat{c})+\lambda \int_{0}^{x} v(x-y) d F_{Y}(y)
$$

if $\hat{c}>\mu$. 
Thus either by (3.22), (3.32) and (3.24) there exists $c^{*} \in\left[0, c_{0}\right]$ such that

$$
0 \leq\left(\mu-c^{*}\right) v_{+}^{\prime}(x)-(\beta+\lambda) v(x)+U\left(c^{*}\right)+\lambda \int_{0}^{x} v(x-y) d F_{Y}(y),
$$

or by (3.23), 3.31) and (3.24) there exists $c_{*} \in\left[0, c_{0}\right]$ such that

$$
0 \leq\left(\mu-c_{*}\right) v_{-}^{\prime}(x)-(\beta+\lambda) v(x)+U\left(c_{*}\right)+\lambda \int_{0}^{x} v(x-y) d F_{Y}(y) .
$$

Therefore, from (3.28), (3.33) and (3.29) and (3.34), we know that either $v_{+}^{\prime}(x)$ satisfies the Hamilton-Jacobi-Bellman equation

$$
\sup _{0 \leq c \leq c_{0}}\left\{(\mu-c) v_{+}^{\prime}(x)-(\beta+\lambda) v(x)+U(c)+\lambda \int_{0}^{x} v(x-y) d F_{Y}(y)\right\}=0,
$$

or $v_{-}^{\prime}(x)$ satisfies Hamilton-Jacobi-Bellman equation

$$
\sup _{0 \leq c \leq c_{0}}\left\{(\mu-c) v_{-}^{\prime}(x)-(\beta+\lambda) v(x)+U(c)+\lambda \int_{0}^{x} v(x-y) d F_{Y}(y)\right\}=0 .
$$

Note that we only know at present that the alternative of these two sentences is true but we do not know if both sentences are true at the same time.

Note that the function $\xi(c):=(\mu-c) v_{+}^{\prime}(x)+U(c)$ is strictly concave in $\left[0, c_{0}\right]$, because the sum of a strictly concave function and a linear function is strictly concave. Since $\zeta$ is strictly concave, it follows that it has only one local maximum. Therefore if (3.35) is satisfied then there exists exactly one limit $c^{*}$ which realizes the supremum in this equation. Similarly the function $\eta(c):=(\mu-c) v_{-}^{\prime}(x)+U(c)$ is strictly concave in $\left[0, c_{0}\right]$. Therefore if $(3.36)$ is satisfied then there exists exactly one limit $c_{*}$ which realizes the supremum in $(3.36)$.

We now prove that $v$ is differentiable. We show that $v_{+}^{\prime}(x)=v_{-}^{\prime}(x)$ in every possible case. First we consider again the inequalities 3.22 3.24). As mentioned above, we do not know which of them is satisfied and how many values of $\tilde{c}$ there are. Note that all values $\tilde{c}$ (if any) realize the supremum in (3.35) or 3.36). We proved above that there is exactly one value which realizes the supremum in these equations (because of strict concavity of $\xi$ and $\eta$ ). Therefore there exists at most three values of $\tilde{c}: \tilde{c}_{1}<\mu$ or $\tilde{c}_{2}>\mu$ or $\tilde{c}_{3}=\mu$. We also know that at least one of these three values exists.

Similarly considering inequalities (3.31), (3.32) and (3.24) we conclude that at least one of at most three values of $\hat{c}: \hat{c}_{1}<\mu$ or $\hat{c}_{2}>\mu$ or $\hat{c}_{3}=\mu$, realizes the supremum in (3.35) or (3.36).

At the beginning we suppose that there exists exactly one $\tilde{c}$ and exactly one $\hat{c}$. Now we consider all possible cases: 
I. $\tilde{c}_{1}<\mu \wedge \hat{c}_{2}>\mu$. In this case (3.22) and (3.32) are satisfied. This means that the supremum in $(3.35)$ is attained at $\tilde{c}_{1}$ and $\hat{c}_{2}$. Since the supremum in (3.35) may be attained at exactly one point, we get $\tilde{c}_{1}=\hat{c}_{2}$, a contradiction. Thus this case is impossible.

II. $\tilde{c}_{2}>\mu \wedge \hat{c}_{1}<\mu$. In this case, (3.23) and (3.31) are satisfied. This means that the supremum in (3.36) is attained at $\tilde{c}_{2}$ and $\hat{c}_{1}$. Since that supremum may be attained at exactly one point, we get $\tilde{c}_{2}=\hat{c}_{1}$, a contradiction. Thus this case is also impossible. Note that excluding cases I and II leads to the conclusion that $v_{+}^{\prime}(x)$ satisfies (3.35) and $v_{-}^{\prime}(x)$ satisfies (3.36) (so now we know that both equations are satisfied).

III. $\tilde{c}_{1}<\mu \wedge \hat{c}_{1}<\mu$. This case was considered in Theorem 3.2 (see (3.13). We proved that $v_{+}^{\prime}(x)=v_{-}^{\prime}(x)$ in that case.

IV. $\tilde{c}_{2}>\mu \wedge \hat{c}_{2}>\mu$. The proof of this case is the same as in case III and leads to $v_{+}^{\prime}(x)=v_{-}^{\prime}(x)$.

V. $\tilde{c}_{1}<\mu \wedge \hat{c}_{3}=\mu$. In this case, (3.22) and (3.24) are satisfied. This means that the supremum in 3.35 is attained at $\tilde{c}_{1}$ and $\mu$. Since that supremum may be attained at exactly one point, we get $\tilde{c}_{1}=\mu$, a contradiction. Thus this case is impossible.

VI. $\tilde{c}_{2}>\mu \wedge \hat{c}_{3}=\mu$.

VII. $\tilde{c}_{3}=\mu \wedge \hat{c}_{1}<\mu$.

VIII. $\tilde{c}_{3}=\mu \wedge \hat{c}_{2}>\mu$.

Cases VI-VIII cannot hold for the same reasons as in case V.

IX. $\tilde{c}_{3}=\mu \wedge \hat{c}_{3}=\mu$. In this case, (3.24) is satisfied. But this means that the supremum in 3.35 and 3.36 is attained at $\mu$. From the definition of local maximum it follows that

$$
U(\mu) \geq(\mu-c) v_{+}^{\prime}(x)+U(c), \quad U(\mu) \geq(\mu-c) v_{-}^{\prime}(x)+U(c)
$$

for all $c$ in some neighborhood of $\mu$. Since $U$ is concave, its left and right derivatives exist. Consequently, the above inequalities give

$$
U_{+}^{\prime}(\mu) \leq v_{+}^{\prime}(x) \leq U_{-}^{\prime}(\mu), \quad U_{+}^{\prime}(\mu) \leq v_{-}^{\prime}(x) \leq U_{-}^{\prime}(\mu) .
$$

Since $U$ is differentiable at $\mu$, we have $v_{+}^{\prime}(x)=v_{-}^{\prime}(x)$ in this case.

We have considered all possible cases when there exists exactly one $\tilde{c}$ and exactly one $\hat{c}$, and found that $v_{+}^{\prime}(x)=v_{-}^{\prime}(x)$ in each case. Assume now that either there exists more than one value of $\tilde{c}: \tilde{c}_{1}<\mu$ or $\tilde{c}_{2}>\mu$ or $\tilde{c}_{3}=\mu$, or there exists more than one value of $\hat{c}$ : $\hat{c}_{1}<\mu$ or $\hat{c}_{2}>\mu$ or $\hat{c}_{3}=\mu$. This also leads to a contradiction because there will be two or more different values which realize the supremum in 3.35 or 3.36 . We know that this is impossible.

Thus we have proved that $v_{+}^{\prime}(x)=v_{-}^{\prime}(x)$ for each possible case. This also means that the value $c^{*}$ (that realizes the supremum in (3.35) and $c_{*}$ (that 
realizes the supremum in (3.36) ) are equal: $c^{*}=c_{*}$. Summarizing, we have proved that there exists exactly one optimal value $c^{*}$ that simultaneously realizes the suprema in (3.35) and in (3.36), and the function $v$ is differentiable and satisfies the Hamilton-Jacobi-Bellman equation

$$
\sup _{0 \leq c \leq c_{0}}\left\{(\mu-c) v^{\prime}(x)-(\beta+\lambda) v(x)+U(c)+\lambda \int_{0}^{x} v(x-y) d F_{Y}(y)\right\}=0
$$

if $c_{0}>\mu$. From (3.37), since $v$ and $U$ are continuous, it follows that $v$ is continuously differentiable if $c^{*} \neq \mu$. We have proved that $v(x)$ is differentiable even at points $x_{0}$ for which $c^{*}=\mu$. Note that the derivative of a differentiable function never has a jump or a removable discontinuity. Moreover, from (2.2) it follows that $v^{\prime}(x)$ is bounded, and so cannot have an infinite discontinuity. However, we cannot exclude that $\lim _{x \rightarrow x_{0}-} v^{\prime}(x)$ or $\lim _{x \rightarrow x_{0}+v^{\prime}(x) \text { does not }}$ exist for $x_{0}$ for which $c^{*}=\mu$, and $v^{\prime}(x)$ has an essential discontinuity at $x_{0}$ for which $c^{*}=\mu$.

From the fact that $v$ is differentiable and from inequality 2.2 we have the following remark.

REMARK 3.4. The function $v^{\prime}$ is bounded and satisfies

$$
0 \leq v^{\prime}(x) \leq \frac{U\left(c_{0}\right)}{\mu \beta}(\lambda+\beta) .
$$

From now on we assume that $U$ is differentiable and the Inada conditions are satisfied:

$$
\lim _{x \rightarrow 0} U^{\prime}(x)=\infty \text { and } \lim _{x \rightarrow \infty} U^{\prime}(x)=0 .
$$

Then we may explicitly calculate the optimal strategy $c^{*}$.

REMARK 3.5. If $U$ is differentiable and satisfies the Inada conditions, then the optimal $c^{*}$ in 3.16 is given by

$$
c^{*}(x)= \begin{cases}\left(U^{\prime}\right)^{-1}\left(v^{\prime}(x)\right) & \text { if }\left(U^{\prime}\right)^{-1}\left(v^{\prime}(x)\right)<c_{0}, \\ c_{0} & \text { if }\left(U^{\prime}\right)^{-1}\left(v^{\prime}(x)\right) \geq c_{0} .\end{cases}
$$

Note that $c^{*} \geq 0$. Indeed, from the Inada conditions we have $\left(U^{\prime}\right)^{-1}(0)$ $=\infty$ and $\left(U^{\prime}\right)^{-1}(\infty)=0$, and from the strict concavity of $U$ we know that $U^{\prime}$, and hence $\left(U^{\prime}\right)^{-1}$, is strictly decreasing in $(0, \infty)$. Moreover $v$ is increasing. Hence $\left(U^{\prime}\right)^{-1}\left(v_{x}(x)\right) \geq 0$ for all $x \geq 0$. Therefore $c^{*} \in\left[0, c_{0}\right]$. This leads to the next remark.

REMARK 3.6. If $U$ is differentiable and satisfies the Inada conditions, then the optimal dividend strategy $\left(c_{t}^{*}\right)_{t \geq 0}$ is given by

$$
c_{t}^{*}= \begin{cases}c^{*}\left(X_{t}\right) & \text { for } 0 \leq t<\tau, \\ 0 & \text { for } t \geq \tau,\end{cases}
$$

where $c^{*}$ is defined in 3.38 . 
TheOREM 3.7. Suppose that $\psi$ is a continuously differentiable, increasing, bounded, and nonnegative solution to (3.3) or (3.16). Then $\psi(x)=v(x)$, and an optimal dividend policy is given by the Markovian strategy $c_{t}^{*}$ defined in 3.39 ).

Proof. This proof is based on the proof of [1, Proposition 2.13]. The function $\psi$ is nonnegative and continuously differentiable in $\mathbb{R}_{\geq 0}$ as a solution of (3.3) or (3.16). Since $e^{-\beta t} \psi(x)$ is continuously differentiable in $\mathbb{R}_{\geq 0}$, using the change of variables formula for finite variation processes (see for instance [9]) we can write

$$
\begin{gathered}
\psi\left(X_{t}\right) e^{-\beta t}-\psi(x)=\int_{0}^{t} \psi^{\prime}\left(X_{s^{-}}\right) e^{-\beta s} \mu d s-\beta \int_{0}^{t} \psi\left(X_{s^{-}}\right) e^{-\beta s} d s \\
-\int_{0}^{t} \psi^{\prime}\left(X_{s^{-}}\right) e^{-\beta s} d C_{s}+\sum_{\substack{X_{s-} \neq X_{s} \\
s \leq t}}\left(\psi\left(X_{s}\right)-\psi\left(X_{s^{-}}\right)\right) e^{-\beta s} .
\end{gathered}
$$

Since $C_{t}=\int_{0}^{t} c_{s} d s$, we have

$$
\int_{0}^{t} \psi^{\prime}\left(X_{s^{-}}\right) e^{-\beta s} d C_{s}=\int_{0}^{t} \psi^{\prime}\left(X_{s^{-}}\right) e^{-\beta s} c_{s} d s .
$$

On the other hand, $X_{s} \neq X_{s^{-}}$only at the arrival of a claim, so by the compensation formula,

$$
\begin{aligned}
M_{t}= & \sum_{\substack{X_{s-} \neq X_{s} \\
s \leq t}}\left(\psi\left(X_{s}\right)-\psi\left(X_{s^{-}}\right)\right) e^{-\beta s} \\
& -\lambda \int_{0}^{t} e^{-\beta s} \int_{0}^{\infty}\left(\psi\left(X_{s^{-}}-y\right)-\psi\left(X_{s^{-}}\right)\right) d F_{Y}(y) d s
\end{aligned}
$$

is a martingale with zero expectation because

$$
0 \leq \psi\left(X_{s}\right) \leq \max _{y \in[0, x+\mu t]} \psi(y)<\infty
$$

for $s \leq t$. The upper bound comes from the fact that $\psi$ is bounded and $X_{t} \leq x+\mu t$. Therefore, we can combine $3.40-3.42$ to obtain

$$
\begin{aligned}
& \psi\left(X_{t}\right) e^{-\beta t}-\psi(x)=\int_{0}^{t} \psi^{\prime}\left(X_{s^{-}}\right) e^{-\beta s} \mu d s-\beta \int_{0}^{t} \psi\left(X_{s^{-}}\right) e^{-\beta s} d s+M_{t} \\
& \quad-\int_{0}^{t} \psi^{\prime}\left(X_{s^{-}}\right) e^{-\beta s} c_{s} d s+\lambda \int_{0}^{t} e^{-\beta s} \int_{0}^{\infty}\left(\psi\left(X_{s^{-}}-y\right)-\psi\left(X_{s^{-}}\right)\right) d F_{Y}(y) d s \\
& =\int_{0}^{t}\left(\psi^{\prime}\left(X_{s^{-}}\right)\left(\mu-c_{s}\right)-(\beta+\lambda) \psi\left(X_{s^{-}}\right)+\lambda \int_{0}^{\infty} \psi\left(X_{s^{-}}-y\right) d F_{Y}(y)\right) e^{-\beta s} d s+M_{t} .
\end{aligned}
$$


Since $\psi$ satisfies $(3.3)$ or $(3.16)$, for every strategy $\left(c_{s}\right)_{s \geq 0}$ we have

$$
\psi\left(X_{t}\right) e^{-\beta t}-\psi(x) \leq-\int_{0}^{t} U\left(c_{s}\right) e^{-\beta s} d s+M_{t} .
$$

Taking the expected values of both sides gives

$$
\psi(x) \geq \mathbb{E}_{x}\left(\psi\left(X_{t}\right) e^{-\beta t}+\int_{0}^{t} U\left(c_{s}\right) e^{-\beta s} d s\right) .
$$

Because $\psi(x)$ is bounded, $\lim _{t \rightarrow \infty} \mathbb{E}_{x}\left(\psi\left(X_{t}\right) e^{-\beta t}\right)=0$. By the bounded convergence theorem,

$$
\psi(x) \geq \mathbb{E}_{x}\left(\int_{0}^{\infty} U\left(c_{s}\right) e^{-\beta s} d s\right)=\mathbb{E}_{x}\left(\int_{0}^{\tau} U\left(c_{s}\right) e^{-\beta s} d s\right)=v_{c}(x),
$$

where in the last equation we have used the fact that $U(0)=0$ and $\left(c_{s}\right)_{s \geq 0}$ is admissible, which means that there are no dividends after ruin occurs $\left(c_{t}=0\right.$ for $\left.t \geq \tau\right)$. Since $\psi(x) \geq v_{c}(x)$ for every strategy $\left(c_{s}\right)_{s \geq 0}$, it follows that $\psi(x) \geq v(x)$. If we take $\left(c_{t}^{*}\right)_{t \geq 0}$ defined in (3.39), then equality holds in (3.43)-(3.45). Because $\psi(x)=v_{c^{*}}(x)$, it follows that $\psi(x) \leq v(x)$. This completes the proof.

Acknowledgements. This work was partially supported by the Ministry of Science and Higher Education of Poland under grant 2011/01/B/HS4 /00982 (2012-2013).

The research of Sebastian Baran was supported by the Faculty of Finance and Law, Cracow University of Economics, under grant no. 158/WF-KM/02/ 2016/M/6158 for the research of young scientists.

\section{References}

[1] P. Azcue and N. Muler, Stochastic Optimization in Insurance: A Dynamic Programming Approach, Springer, 2014.

[2] J. Eisenberg and H. Schmidli, Minimising expected discounted capital injections by reinsurance in a classical risk model, Scandinavian Actuarial J. 3 (2011), 155-176.

[3] B. De Finetti, Su un'impostazione alternativa della teoria collettiva del rischio, Trans. XV Intern. Congress of Actuaries, Vol. 2, 1957, 433-443.

[4] H. U. Gerber, Introduction to Mathematical Risk Theory, Richard D. Irwin, 1979.

[5] G. H. Hardy, Some results concerning the behaviour at infinity of a real and continuous solution of an algebraic differential equation of the first order, Proc. London Math. Soc. 10 (1912), 451-468.

[6] F. Hubalek and W. Schachermayer, Optimizing expected utility of dividend payments for a Brownian risk process and a peculiar nonlinear ODE, Insurance Math. Econom. 34 (2004), 193-225.

[7] N. Kulenko and H. Schmidli, Optimal dividend strategies in a Cramér-Lundberg model with capital injections, Insurance Math. Econom. 43 (2008), 270-278. 
[8] V. Marić, Asymptotic behavior of solutions of a nonlinear differential equation of the first order, J. Math. Anal. Appl. 38 (1972), 187-192.

[9] P. Protter, Stochastic Integration and Differential Equations, Springer, Berlin, 1992.

[10] T. Rolski, H. Schmidli, V. Schmidt and J. Teugles, Stochastic Processes for Insurance and Finance, Wiley, New York, 1999.

[11] H. Schmidli, Stochastic Control in Insurance, Springer, London, 2008.

[12] S. Thonhauser and H. Albrecher, Optimal dividend strategies for a compound Poisson process under transaction costs and power utility, Stochastic Models 27 (2011), 120140.

[13] R. L. Wheeden and A. Zygmund, Measure and Integral. An Introduction to Real Analysis, Dekker, New York, 1977.

Sebastian Baran

Department of Mathematics

Cracow University of Economics

31-510 Kraków, Poland

E-mail: sebastianbaran13@gmail.com
Zbigniew Palmowski Faculty of Pure and Applied Mathematics Wrocław University of Science and Technology 50-370 Wrocław, Poland E-mail: zbigniew.palmowski@gmail.com 
